\title{
UV and EUV Instruments
}

January 2010

to appear in:

Landolt-Börnstein, New Series VI/4A, Astronomy, Astrophysics, and Cosmology; Instruments and Methods, ed. J.E. Trümper, Springer-Verlag, Berlin, 2010

\section{K. Werner}

Institut for Astronomy and Astrophysics

Kepler Center for Astro and Particle Physics

University of Tübingen

Sand 1, 72076 Tübingen, Germany 


\section{$1.4 \quad$ UV and EUV instruments}

We describe telescopes and instruments that were developed and used for astronomical research in the ultraviolet (UV) and extreme ultraviolet (EUV) regions of the electromagnetic spectrum. The wavelength ranges covered by these bands are not uniquely defined. We use the following convention here: The EUV and UV span the regions $\lambda \lambda 100-912 \AA$ and $912-3000 \AA$, respectively. The limitation between both ranges is a natural choice, because the hydrogen Lyman absorption edge is located at $\lambda 912 \AA$. At smaller wavelengths, astronomical sources are strongly absorbed by the interstellar medium. It also marks a technical limit, because telescopes and instruments are of different design. In the EUV range, the technology is strongly related to that utilized in $\mathrm{X}$-ray astronomy, while in the UV range the instruments in many cases have their roots in optical astronomy. We will, therefore, describe the UV and EUV instruments in appropriate conciseness and refer to the respective chapters of this volume for more technical details.

The $\lambda 100 \AA$ limit of the EUV range to the soft X-ray range is fuzzy, because it is covered by both, specific X-ray and EUV instrumentation, and because there is no scientific motivation for a sharp distinction between these regions. As a consequence, there will be some minor overlap with the X-ray chapter of this volume concerning the presentation of specific instruments. In contrast, the long-wavelength limit of the UV range to the optical region is an obvious choice. The $\lambda>3000 \AA$ region is accessible with ground-based telescopes, whereas investigation shortwards of this limit requires observations from above Earth's atmosphere.

All facts presented here are mainly taken from original papers referred to in Table 1. A number of reviews are useful sources, e.g.: [69Wil] and [72Ble] on UV astronomy, [71Car] on electronic imaging devices, [91Bob] on the cosmic far-UV $(\lambda \lambda$ 1000-2000 $\AA)$ background, and [00Bow] on EUV astronomy. Significant books on the state-of-the-art in the relatively concise field of EUV astronomy (in comparison to the UV) are two conference proceedings [91Mal], [96Bow], and a very useful, exhaustive presentation by [03Bar].

We restrict our description here to space-based instruments, i.e., to those facilities that were carried by satellites or by interplanetary and lunar probes. For conciseness, we do not address the issue of sounding rocket experiments, although they have been essential for the development of UV and EUV instruments and obtaining early scientific results. Likewise, we will not describe stratospheric balloon experiments, which exploit the fact that Earth's atmosphere beyond a height of $\approx 40 \mathrm{~km}$ above sea level is sufficiently transparent in the $\lambda \lambda 2000-3000 \AA$ region to perform astronomical observations.

For UV and EUV instrumentation used to observe the Sun, we refer to another chapter of this volume. Solar observatories (e.g. SOHO, STEREO) occasionally performed stellar observations for calibration purposes [07Val]. We also disregard here UV/EUV instruments that were used by space probes to observe exclusively solar-system objects (Moon, planets, interplanetary matter, etc.). But we will point out UV/EUV instruments of such probes that were also used to perform astronomical observations of Galactic and extragalactic objects.

In the following, we describe EUV and UV instruments in two separate sections. In the sense just outlined, Table 1 represents a complete compilation of all EUV and UV instruments that flew on space probes, in chronological order of their launch date. For details on the instrumentation references are made to Table 2 .

Most instruments were carried on Earth-orbiting satellites, the first few of them were unstabilized. Important measurements were contributed from instruments that flew on missions to planets. During interplanetary cruise phases, astronomical observations were performed with instruments primarily devoted to the study of planetary atmospheres: Venera $2 \& 3$ to Venus, Mariner 9 to Mars (and in Mars orbit), and Voyager $1 \& 2$ to the outer solar system. Other instruments were operated on manned space vehicles: Gemini (during extravehicular stand-up activities by astronauts), Apollo (on the lunar surface and during translunar coast), Soyuz, the MIR and Skylab space stations, and quite frequently on several Space Shuttle flights. 


\subsubsection{EUV instruments}

An excellent summary about the current state of technology for EUV space-instrumentation is given in [03Bar].

\subsubsection{EUV detectors}

EUV detector developments were determined by two approaches (photon counting devices):

- Proportional counters (as used in X-ray astronomy), and

- Photomultipliers (as used in UV range).

The problem with proportional counters is, that detector windows should not absorb photons (this is a much smaller problem in the X-ray range). Therefore, thinner windows or plastic windows are used. But significant gas losses occur, hence, "thin window, gasflow" proportional counters were developed. But these are limited to $\lambda<200 \AA$, therefore other approaches are more common in EUV astronomy: CEM (channel electron multiplier) and MCP (microchannel plates). The CEM is a compact, windowless version of a photomultiplier. Therefore, photons at all EUV wavelengths can be detected.

Two-dimensional (2-d) position-sensitive detectors are required for observations with focussing instruments and for an efficient recording of spectra. Three techniques have been advanced:

- MCP detectors (e.g. used in Chandra/LETG, EUVE, ROSAT/WFC, EXOSAT/CMA, Einstein/HRI). Essentially, MCPs are arrays of single CEMs. They have no energy resolution.

- IPCs (imaging proportional counters; e.g. ROSAT/PSPC). These are position sensitive proportional counters (PSPC). They have rather modest energy resolution.

- CCDs (charge coupled devices); solid-state semiconductor detectors developed for X-ray instruments (XMM-Newton). They are sensitive up to $\lambda \approx 100 \AA$ and have rather modest energy resolution in the EUV. They are different from "usual" CCDs (i.e. those recording optical photons) which were often used in UV/EUV experiments in combination with UV-optical converters (so-called intensified CCDs, see below).

In EUV experiments, MCPs are mostly used because of the limited wavelength range of proportional counters. Quantum efficiencies of MCPs are relatively low $(\approx 5 \%)$. They can be increased significantly by depositing a photocathode on their front face. The materials are mostly alkali-halides (e.g. $\mathrm{MgF}_{2}$, CsI, KBr). Different read-out systems for 2-d MCPs are used, e.g., a wedge-and-strip anode (WSA), the mostly used design (e.g. EUVE). MCPs are solar blind.

\subsubsection{EUV telescopes}

The first orbiting EUV telescopes (after Apollo-Soyuz/EUVT) were grazing-incidence telescopes of Wolter types I and II (EUVE, ROSAT-WFC). Nested mirrors increase the collecting area (type I Wolter). Grazing-incidence is necessary for wavelengths below $\lambda \approx 300 \AA$. However, the incidence angles can be rather large $\left(10^{\circ}\right)$ compared to X-ray telescopes $\left(1-2^{\circ}\right)$, because the reflectivity is still quite high in EUV. Likewise, the tolerances on surface roughness are not so severe as for $\mathrm{X}$-rays. Materials are usually glass or metal shells coated with gold.

At wavelengths larger than $\lambda \approx 500 \AA$ normal-incidence telescopes with relatively high reflectivity by $\mathrm{SiC}$ are possible. Aluminum coated with $\mathrm{LiF}$ or $\mathrm{MgF}_{2}$ is preferred above $\lambda \lambda 1050 \AA$ and $1150 \AA$, respectively (e.g. ORFEUS, HUT, HST, FUSE). 
More recently, normal-incidence telescopes are used even at short wavelengths, i.e., $\lambda<300 \AA$. They use interference techniques for reflection with multilayer coatings of optical surfaces (ALEXIS, 6 different telescopes with large collecting area). The price to pay is a narrow bandpass. Its width is about $10 \%$ of the peak wavelength.

Simple mechanical collimators instead of imaging telescopes were employed in a number of EUV missions that particularly aimed at measuring the Galactic EUV background radiation and diffuse emission from the interstellar medium (e.g. CHIPS, EURD), but also for stellar observations (e.g. Voyager/UVS).

\subsubsection{EUV photometry and spectroscopy}

Thin film filters and optical coating materials define bandpasses for photometry and block unwanted background radiation. There are two categories of filters:

- Plastic films; material: hydrocarbon polymers (polypropylene, parylene, Lexan), and

- Metal foils (often used with mechanical support grids).

Spectroscopic instruments in the EUV utilize reflection (EUVE, CHIPS) and transmission gratings (Chandra/LETG, EXOSAT/TGS). Filters can suppress higher-order spectra.

\subsubsection{Notes on specific EUV missions}

For a long time, it was thought that observations in the EUV band would be impossible, because of the presumed high opacity of the interstellar medium [59All]. Encouraged by new evidence on the average density of neutral hydrogen and probable inhomogeneities, a first successful search for EUV sources was performed in 1975 with an instrument on the Apollo-Soyuz Test Project [77Bow]. Further detections were made in the soft-energy range of X-ray observatories (Einstein, EXOSAT). Important milestones were the photometric all-sky surveys conducted with the wide-field camera (WFC) aboard ROSAT and with the EUVE satellite. For the first (and hitherto the only) time, EUVE offered to a wide astronomical community access to a spectroscopic instrument covering the entire EUV range. ALEXIS is remarkable and probably pathbreaking, because it utilized for the first time a normal-incidence telescope in the far-EUV range.

A few UV instruments were also capable of performing near-EUV spectroscopy, e.g., HUT, ORFEUS/BEFS, and the UVS instruments on the Voyager probes. At the time of writing (2009), the UVS on Voyager 1 is still operating, more than 30 years after launch. It stares at a point in anti-sun direction and returns spectra of the interplanetary medium. These are used to study the Ly $\alpha$ emission from interstellar gas penetrating the solar system [09Hol]. Currently, there is no EUV instrument in operation, and there are no accepted proposals for future EUV missions. The XMM-Newton and Chandra X-ray observatories provide access to the very-far $\operatorname{EUV~}(\lambda<150 \AA)$, only. 


\subsubsection{UV instruments}

A concise overview about the current state of technology for UV space-instrumentation and possible future developments is given in a special section of [99Mor].

\subsubsection{UV detectors}

In the first instruments, and up to the mid-1980s, photomultipliers were used for photometric work. Before the availability of 2-d detectors, spectra were scanned with photomultipliers, too (e.g. OAO-2, Copernicus, Apollo 17, Astron).

In some instruments (OAO-2, Copernicus, IUE) television (TV) camera tubes were used as 2 -d detectors. The secondary electron conduction (SEC) vidicon detectors aboard IUE integrated spectrographic images on a potassium chloride SEC target and were then read out by scanning the SEC target with an electron beam. The photocathode of the TV camera tubes, designed for visible light response, required a converter (with a CsTe photocathode) to transform UV into visible radiation.

Image recording on photographic film was an important detection technique for a long time. Two primary methods were used: one is electronography, in which high-energy electrons from the photocathode cause the blackening of the grains directly; the other makes use of a phosphor screen to convert the electron energy into visible light, which is then recorded on ordinary, lightsensitive photographic film. In the latter method the electronic imaging device serves mainly to intensify the image, so as to increase the speed of the ordinary photographic process, and, in some cases, to extend its spectral range into wavelength ranges to which the film is not directly sensitive. Hence, these latter type devices are commonly called image intensifiers or image converters [71Car]. Film detectors were exclusively used in short-term, American and Soviet manned space missions, commencing with Gemini flights in 1966, and ending with several instruments on Space Shuttles, up to 1995 (UIT, FUVIS).

Today, MCPs as well as CCDs (the latter at wavelengths longer than $\lambda \approx 1500 \AA$ ) are utilized. The UV response of CCDs is sometimes enhanced by addition of a phosphor, such as Lumogen on the HST/WFPC-2 CCDs. CCDs with improved UV response are under active development. Intensified CCDs (ICCD) are image intensifier tubes or MCPs in front of optically sensitive CCDs, e.g., MCP-intensified CCDs in the Optical Monitor (OM) of XMM-Newton. The first use of an MCP detector was on the Voyager UV-Spectrographs; they served as image intensifiers for a newly developed self-scanned linear anode array (so-called Ssanacon) [77Bro].

Another detector type are intensified photodiode arrays. One realisation was an MCP with a phosphor screen anode put in front of a Reticon, that is, a visual-photon recording photodiode array (HUT). Digicons were used in the GHRS and FOS spectrographs aboard HST. They consist of a photocathode, magnetic and electrostatic focussing coils and electrodes, and a diode array. The photoelectrons are accelerated directly onto the diodes with no conversion into visible-light photons. Digicons gave way after the first-generation of HST instruments to either multi-anode microchannel arrays (MAMAs, HST/STIS instrument) which achieve small pixels with high photon-count rates, or electron-bombarded CCDs.

\subsubsection{UV telescopes}

Normal-incidence mirrors are used. As for the coatings of mirrors (and optical elements), different materials are necessary for different wavelength bands in order to achieve high performance. For example, the HST mirror coating $\left(\mathrm{Al}+\mathrm{MgF}_{2}\right)$ is "blind" for $\lambda<1150 \AA$, hence, other materials are needed for high reflectivity when going to the far-UV (towards the hydrogen Lyman edge). 
For example, the FUSE instrument consists of four coaligned telescopes and spectrographs. Two channels with $\mathrm{SiC}$ coatings cover the range $\lambda \lambda$ 905-1100 $\AA$, and two channels with $\mathrm{Al}+\mathrm{LiF}$ coatings cover the range $\lambda \lambda 1000-1195 \AA$ (04Moo).

In earlier instruments refractive optics cameras were employed for imaging (Gemini), and later on Schmidt cameras. Open collimators, i.e., non-imaging instruments, were also used for UVbackground studies since the earliest mission (Kosmos 51) until more recently (EURD). Also, point source detection and spectroscopy with non-imaging instruments were performed (Apollo 17, Voyager $1 \& 2$, IMAPS).

\subsubsection{UV photometry, spectroscopy, and polarimetry}

In analogy to instruments working in the optical wavelength band, filters, coating materials and detector efficiency characteristics define bandpasses for photometry and block unwanted background radiation. Likewise, the principles of employed spectroscopic (prisms, grisms, gratings) and polarimetric devices are in principle the same as in optical astronomy.

\subsubsection{Notes on specific UV missions}

The early-phase history of UV space instruments culminated in 1968 with the launch of OAO-2, the first observatory-type mission. A similar success was OAO-3 (Copernicus), which obtained first high-resolution UV spectra. TD-1A and ANS were important UV-survey instruments. IUE was an outstanding success. For almost two decades it was operated as a true observatory with real-time access by guest observers at ground stations, and delivered more than 100,000 UV spectra of all kinds of sources. FUSE was seminal because it took high-resolution far-UV spectra $(\lambda \lambda 912$ $1180 \AA$ ) for many years. GALEX made a deep UV all-sky survey.

The Hubble Space Telescope (HST) carries a diverse suite of imaging and spectroscopic instruments working in a broad wavelength range spanning from the UV to the infrared. It is the only orbiting space telescope that was frequently maintained by Space Shuttle crews repairing instruments and installing new ones. In 2004, HST lost its UV spectroscopic capability when the STIS spectrograph failed. Hence, at the time of writing (2009), no UV spectroscopic instrument is available at all (except for the small UV/optical monitors aboard the XMM-Newton and Swift X-ray observatories). For 2009, the last of HST servicing missions is planned, during which STIS shall be repaired and a new UV spectrograph (COS) shall be installed. If successful, then HST will again provide access to the UV for another $\approx 5$ years.

There are no accepted proposals for spectroscopic UV missions beyond HST, except for a Russian-led multi-national initiative, the World Space Observatory Ultraviolet (WSO/UV). It shall perform imaging and spectroscopy in the $\lambda \lambda$ 1020-3200 $\AA$ band (resolving power $\mathrm{R}=1000$ and 50000 ) and is planned for launch in 2014. Two Indian-led multiple-band UV-imaging missions (Astrosat/UVIT and GSat4/TAUVEX) are scheduled for launch in 2009. 
Table 1. List of space-based UV and EUV instruments. Abbreviations: ph.=photometry, im.=imaging, sp.=spectroscopy. The numbers in the column "Instr." refer to the description of telescopes, instruments, and detectors in Table $2 \Delta \lambda$ and $\mathrm{R}$ denote spectral resolution and resolving power, respectively.

\begin{tabular}{|c|c|c|c|c|c|c|c|}
\hline & Mission/Instrument & $\begin{array}{l}\text { Country } \\
\text { or Agency }\end{array}$ & Period & Instr. & $\begin{array}{c}\text { Wavelength } \\
\text { Range }[\AA]\end{array}$ & Remarks & Ref. \\
\hline & Kosmos 51 & USSR & 1964 & 1,7 & $2300-7500$ & 2 bands, UV background & 70Di1,72Dim \\
\hline & $196483 \mathrm{C}$ & USA & 1964 & 2,7 & $1300-1650$ & 1 band, stars & $67 \mathrm{Smi}$ \\
\hline & Venera 2,3 & USSR & 1965 & 1,4 & $1050-1340$ & 2 bands, UV background & $67 \mathrm{Kur}, 68 \mathrm{Kur}$ \\
\hline & Gemini $10-12 / \mathrm{S}-013$ & USA & 1966 & $3,6,16$ & $2500-4400$ & $\Delta \lambda=7-20 \AA$, stars & $70 \mathrm{Kon}, 74 \mathrm{Spe}$ \\
\hline & Kosmos 213 & USSR & 1968 & 1,7 & $2000-6500$ & 2 bands, UV background & 70Di1,72Dim \\
\hline & Kosmos 215 & USSR & 1968 & 1,7 & $1250-2800$ & several telescopes/bands, stars & 70Di2,76Dim \\
\hline & OAO-2 & USA & $1968-73$ & $3,8,16$ & $1000-4250$ & multiband im., sp.: $\Delta \lambda=12$ and $22 \AA$ & $72 \mathrm{Cod}$ \\
\hline & Mariner 9/UVS & USA & 1971 & $2,7,17$ & $1150-3400$ & $\Delta \lambda=7.5$ and $15 \AA$ & 71Pea,75Mol \\
\hline & Salyut 1 /Orion 1 & USSR & 1971 & $3,6,16$ & $2000-3800$ & $\mathrm{R}=500$ & $72 \mathrm{Gur}$ \\
\hline & STP 72-1 & USA & 1972 & 1,10 & $912-1050$ & 1 band, UV background & 84Opa \\
\hline & Apollo 16/S-201 & USA & 1972 & $3,6,14$ & $1050-1600$ & 2 bands, operated on lunar surface & 73Car,83Car \\
\hline & Apollo 17/S-169 & USA & 1972 & $1,7,17$ & $1180-1680$ & $\Delta \lambda=10 \AA$ & 73Fas, 75 Hry \\
\hline & Copernicus (OAO-3) & USA & $1972-81$ & $3,7,17$ & $912-3275$ & $\Delta \lambda=0.05-0.4 \AA$ & $73 \operatorname{Rog}$ \\
\hline & TD-1A & ESRO & $1972-74$ & $3,7,17$ & $1350-2550$ & S2/68 instr. sp. $\Delta \lambda=35-40 \AA$, ph. $2750 \pm 310 \AA$ & $73 \mathrm{Bok}$ \\
\hline & & & & $3,7,17$ & $2000-2900$ & S59 instr. $\Delta \lambda=1.7 \AA$ & 74Jag \\
\hline & Soyuz 13 /Orion 2 & USSR & 1973 & $3,6,15$ & $2000-4000$ & $\Delta \lambda=8-28 \AA$ & $76 \mathrm{Gur}$ \\
\hline & Skylab 2-4 & USA & 1973-74 & $3,6,15$ & $1300-5000$ & S-019: $\Delta \lambda=2-42 \AA$ & $75 \mathrm{Hen}, 77 \mathrm{Oca}$ \\
\hline & & & & $3,6,18$ & $2200-3000$ & S-183: one band, French instrument & 77Lag,88Vui \\
\hline & & & & $3,6,14$ & $1100-1500$ & S-201: one band & $74 \mathrm{Car}$ \\
\hline & ANS & NL & $1974-76$ & $3,7,17$ & $1500-3250$ & multiple bands & 75Dui, $82 \mathrm{Wes}$ \\
\hline & D2B-Aura/ELZ & $\mathrm{F}$ & 1975 & $3,7,16$ & $1100-3300$ & multiple bands, UV background & $78 \mathrm{Mau}$ \\
\hline & Apollo-Soyuz/EUVT & USA,USSR & 1975 & 3,10 & $40-1550$ & multiple bands, 1st EUV source detections & 77Bow,80Par \\
\hline & Solrad-11B & USA & 1976 & 1,7 & $1220-1500$ & 1 band, UV background & 83Wel \\
\hline & Prognoz-6/Galaktika & USSR & $1977-78$ & $1,7,17$ & $1100-1850$ & $\Delta \lambda=100 \AA, \mathrm{UV}$ background & $82 Z v e$ \\
\hline \multirow{5}{*}{ 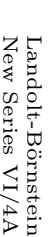 } & Voyager $1+2 /$ UVS & USA & $1977-$ & $1,12,16$ & $500-1700$ & $\Delta \lambda=18 \AA, 1$ st EUV star spectra & 77Bro \\
\hline & Einstein/HRI,OGS & USA & $1978-81$ & $3,9,18$ & $3-120$ & HRI: multiband im., with OGS: sp. $R=10-50$ & $79 \mathrm{Gia}$ \\
\hline & IUE & Intl. & $1978-96$ & $3,8,17$ & $1150-3300$ & $\mathrm{USA}, \mathrm{ESA}, \mathrm{UK} ; \Delta \lambda=0.1$ and $6 \AA$ & 78Bog,97Har \\
\hline & Spacelab 1/VWFC & USA & 1983 & $3,6,14$ & $1250-3000$ & Shuttle flight, 3 bands, French instrument & $94 \mathrm{Tob}$ \\
\hline & Astron & USSR & 1983-89 & $3,7,17$ & $1100-3500$ & $\Delta \lambda=0.4$ and $28 \AA$ & 84Boy,86Boy \\
\hline
\end{tabular}


Table 1. (continued)

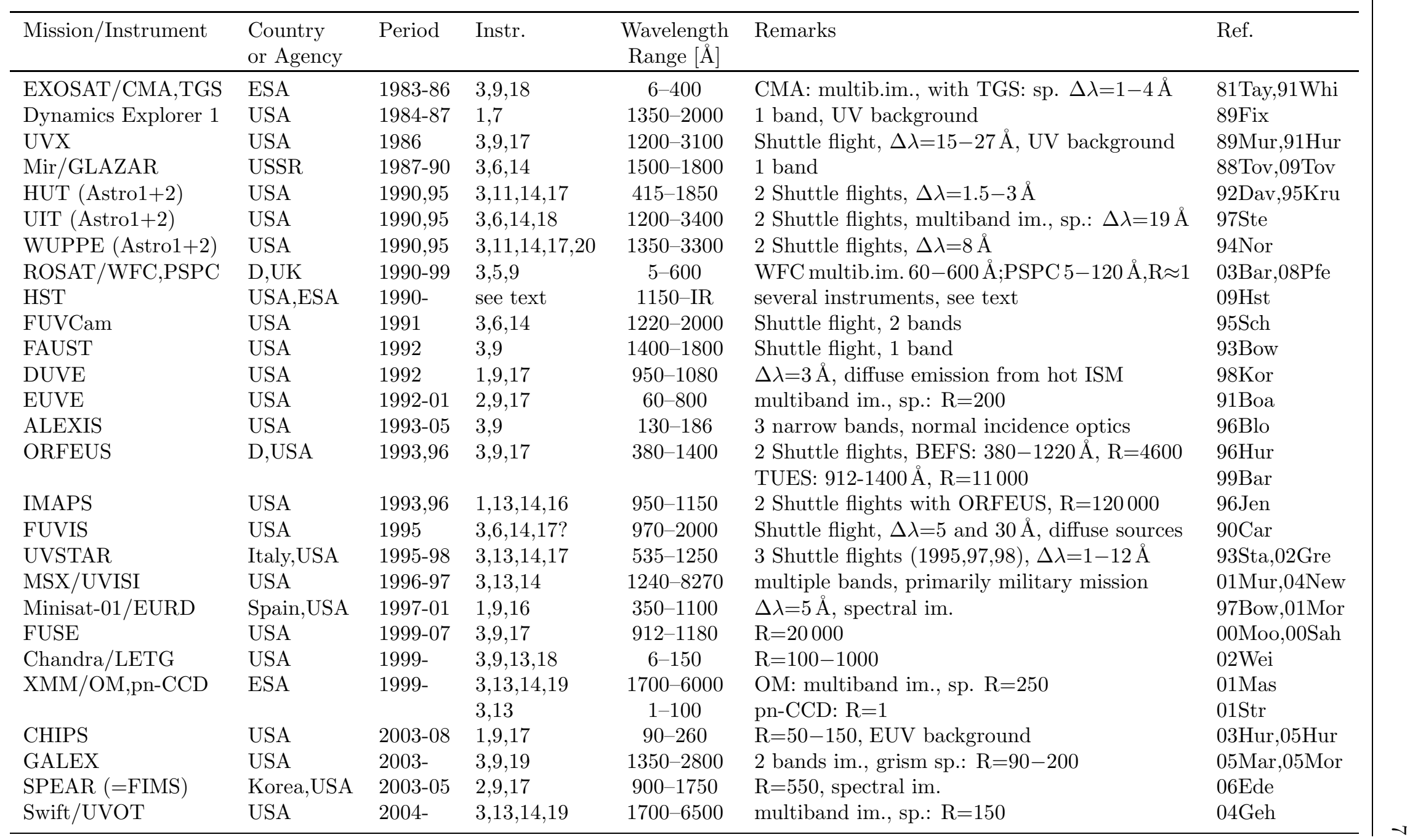


Table 2. Instrumentation reference for UV and EUV space missions listed in Table 1.

\begin{tabular}{lll}
\hline & $\#$ & Description \\
\hline Telescope type & 1 & mechanical collimator \\
& 2 & collecting mirror \\
Detector & 3 & imaging mirror \\
& 5 & Geiger counter \\
& 6 & pas proportional counter \\
7 & photomultiplier \\
8 & television tube (including UV-visible photon converter) \\
9 & microchannel plate (MCP) \\
10 & channel electron multiplier (CEM) \\
11 & photodiode detector (Reticon, Digicon) \\
12 & MCP-intensified self-scanned linear anode array (Ssanacon) \\
13 & charge-coupled device (CCD) \\
14 & image intensifier (e.g. MCP); acts as UV-visible photon converter \\
& in front of optical photon detectors (film, CCDs, photodiodes) \\
& 15 & objective prism \\
16 & objective grating \\
17 & reflection grating \\
18 & transmission grating \\
19 & grism \\
20 & polarizing beamsplitter \\
\hline
\end{tabular}

\subsubsection{References for 1.4}

59All Aller, L.H.: Publ. Astron. Soc. Pacific 71 (1959) 324.

67Smi Smith, A.M.: Astrophys. J. 147 (1967) 158.

67KurKurt, V.G., Sunyaev, R.A.: Soviet J. Exp. Theoret. Ph. Letters 5 (1967) 246.

68Kur Kurt, V.G., Sunyaev, R.A.: Soviet Astron. 11 (1968) 928.

69Wil Wilson, R., Boksenberg, A.: Annu. Rev. Astron. Astrophys. 7 (1969) 421.

70Di1 Dimov, N.A., Severny, A.B., Zvereva, A.M.: in Ultraviolet Stellar Spectra and Ground-Based

Observations, eds. L. Houziaux, Butler, H.-E., IAU Symp. 36 (1970), 325.

70Di2 Dimov, N.A.: in Ultraviolet Stellar Spectra and Ground-Based Observations, eds. L. Houziaux, Butler, H.-E., IAU Symp. 36 (1970), 138.

70KonKondo, Y., Henize, K.G., Kotila, C.L.: Astrophys. J. 159 (1970) 927.

71Car Carruthers, G.R.: Astrophys. Sp. Science 14 (1971) 332.

71Pea Pearce, J.B., Gause, K.A., Mackey, E.F., Kelly, K.K., Fastie, W.G., Barth, C.A.: Appl. Optics 10 (1971) 805.

72Ble Bless, R.C., Code, A.D.: Annu. Rev. Astron. Astrophys. 10 (1972) 197.

72CodCode, A.D. (ed.).: The Scientific Results from the Orbiting Astronomical Observatory (OAO-2), NASA SP-310 (1972).

72DimDimov, N.A., Zvereva, A.M., Severny, A.B.: Izv. Krymskoj Astrofiz. Obs. 45 (1972) 67.

72Gur Gurzadyan, G.A., Ohanesyan, J.B.: Space Sci. Rev. 13 (1972) 647.

73BokBoksenberg, A., Evans, R.G., Fowler, R.G., et al.: Mon. Not. R. Astr. Soc. 163 (1973) 291.

73Car Carruthers, G.R.: Appl. Opt. 12 (1973) 2501.

73Fas Fastie, W.G.: The Moon 7 (1973) 49. 
73RogRogerson, J.B., Spitzer, L., Drake, J.F., et al.: Astrophys. J. Lett. 181 (1973) L97.

74Car Carruthers, G.R., Opal, C.B., Page, T.L., Meier, R.R., Prinz, D.K.: Icarus 23 (1974) 526.

74Jag de Jager, C., Hoekstra, R., van der Hucht, K.A.: Astrophys. Sp. Science 26 (1974) 207.

74Spe Spear, G.G., Kondo, Y., Henize, K.G.: Astrophys. J. 192 (1974) 615.

75Dui van Duinen, R.J., Aalders, J.W.G., Wesselius, P.R., Wildeman, K.J., Wu, C.C., Luinge, W., Snel, D.: Astron. Astrophys. 39 (1975) 159.

75Hen Henize, K.G., Wray, J.D., Parsons, S.B., et al.: Astrophys. J. Lett. 199 (1975) L119.

75Hry Henry, R.C., Weinstein, A., Feldman, P.D., Fastie, W.G., Moss, H.W.: Astrophys. J. 201 (1975) 613.

75Mol Molnar, M.R.: Astrophys. J. 200 (1975) 106.

76DimDimov, N.A., Teres, E.I.: Izv. Krymskoj Astrofiz. Obs. 55 (1976) 196.

76Gur Gurzadyan, G.A., Jarakyan, A.L., Krmoyan, M.N., Kashin, A.L., Loretsyan, G.M., Ohanesyan, J.B.: Space Sci. Rev. 40 (1976) 393.

77BowBowyer, S., Margon, B., Lampton, M., Paresce, F., Stern, R.: Apollo-Soyuz Test Project Summary Science Report (Vol. 1), NASA SP-412 (1977) p. 49.

77Bro Broadfoot, A.L., Sandel, B.R., Shemansky, D.E., et al.: Space Sci. Rev. 21 (1977) 183.

77Lag Laget, M., Saisse, M., Vuillemin, A.: Appl. Opt. 16 (1977) 961.

77Oca Ocallaghan, F.G., Henize, K.G., Wray, J.D.: Appl. Opt. 16 (1977) 973.

78Bog Boggess, A., Carr, F.A., Evans, D.C., et al.: Nature 275 (1978), 372.

78MauMaucherat-Joubert, M., Cruvellier, P., Deharveng, J.M.: Astron. Astrophys. 70 (1978) 467.

79Gia Giacconi, R., Branduardi, G., Briel, U., et al.: Astrophys. J. 230 (1979) 540.

80Par Paresce, F., McKee, C., Bowyer, S.: Astrophys. J. 240 (1980) 387.

81Tay Taylor, B.G., Andresen, R.D., Peacock, A., Zobl, R.: Space Sci. Rev. 30 (1981) 479.

82WesWesselius, P.R., van Duinen, R.J., de Jonge, A.R.W., et al.: Astron. Astrophys. Suppl. Ser. 49 (1982) 427.

82Zve Zvereva, A.M., Severny, A.B., Granitzky, L.V., et al.: Astron. Astrophys. 116 (1982) 312.

83Car Carruthers, G.R., Page, T.: Astrophys. J. Suppl. 53 (1983) 623.

83Wel Weller, C.S.: Astrophys. J. 268 (1983) 899.

84Boy Boyarchuk, A.A., Gershberg, R.E., Granitskij, L.V., et al.: Sov. Astron. Lett. 10 (1984) 67.

84OpaOpal, C.B., Weller, C.: Astrophys. J. 282 (1984) 445.

86Boy Boyarchuk, A.A.: Irish Astron. J. 17 (1986) 352.

88Tov Tovmasyan, G.M., Khodzhayants, Yu.M., Krmoyan M.N., et al.: Sov. Astron. Lett., 14 (1988) 123.

88Vui Vuillemin, A.: Astron. Astrophys. Suppl. Ser. 72 (1988) 249.

89Fix Fix, J.D., Craven, J.D., Frank, L.A.: Astrophys. J. 345 (1989) 203.

89MurMurthy, J., Henry, R.C., Feldman, P.D., Tennyson, P.D.: Astrophys. J. 336 (1989) 954.

90Car Carruthers, G.R., Heckathorn, H.M., Witt, A.N., Raymond, J.C., Opal, C.B., Dufour, R.J.: in The Galactic and Extragalactic Background Radiation, IAU Symp. 139, Reidel, Dordrecht (1990) 459.

91Boa Bowyer, S., Malina, R.F.: Extreme Ultraviolet Astronomy, eds. R.F. Malina, S. Bowyer, Pergamon Press, Elmsford (1991) 397.

91BobBowyer, S.: Annu. Rev. Astron. Astrophys. 29 (1991) 59.

91Hur Hurwitz, M., Bowyer, S., Martin, C.: Astrophys. J. 372 (1991) 167.

91Mal Malina, R.F., Bowyer, S. (eds.): Extreme Ultraviolet Astronomy, Pergamon Press (1991).

91WhiWhite, N.E.: Extreme Ultraviolet Astronomy, eds. R.F. Malina, S. Bowyer, Pergamon Press (1991) 15.

92DavDavidsen, A.F., Long, K.S., Durrance, S.T., et al.: Astrophys. J. 392 (1992) 264.

93BowBowyer, S., Sasseen, T.P., Lampton, M., Wu, X.: Astrophys. J. 415 (1993) 875.

93Sta Stalio, R., Sandel, B.R., Broadfoot, A.L., Chavez, M.: Adv. Space Res. 13 (1993) 379. 
94Nor Nordsieck, K.H., Code, A.D., Anderson, C.M.: X-ray and Ultraviolet Polarimetry, ed. S. Fineschi, Proc. SPIE, 2010 (1994) 2.

94Tob Tobin, W., Viton, M., Sivan, J.-P.: Astron. Astrophys. Suppl. Ser. 107 (1994) 385.

95KruKruk, J.W., Durrance, S.T., Kriss, G.A., et al.: Astrophys. J. 454 (1995) L1.

95Sch Schmidt, E.G., Carruthers, G.R.: Astrophys. J. Suppl. 96 (1995) 605.

96Blo Bloch, J.J.: Astrophysics in the Extreme Ultraviolet, eds. S. Bowyer, R.F. Malina, Kluwer, Dordrecht (1996), 7.

96BowBowyer, S., Malina, R.F. (eds.): Astrophysics in the Extreme Ultraviolet, IAU Coll. 152, Kluwer, Dordrecht (1996).

96Hur Hurwitz, M., Bowyer, S.: Astrophysics in the Extreme Ultraviolet, eds. S. Bowyer, R.F. Malina, Kluwer, Dordrecht (1996), 601.

96Jen Jenkins, E.B., Reale, M.A., Zuccino, P.M., Sofia, U.J.: Astrophys. Space Sci. 239 (1996), 315.

97BowBowyer, S., Edelstein, J., Lampton, M.: Astrophys. J. 485 (1997) 523.

97Har Harris, R.A. (ed.): IUE Spacecraft Operations, Final Report, ESA SP-1215 (1997).

97Ste Stecher, T.P., Cornett, R.H., Greason, M.R.: Publ. Astron. Soc. Pacific 109 (1997) 584.

98Kor Korpela, E.J., Bowyer, S., Edelstein, J.: Astrophys. J. 495 (1998) 317.

99Bar Barnstedt, J., Kappelmann, N., Appenzeller, I., et al.: Astron. Astrophys. Suppl. Ser. 134 (1999) 561.

99MorMorse, J.A., Shull, J.M., Kinney, A.L. (eds.): Ultraviolet-Optical Space Astronomy Beyond HST, ASP Conf. Ser. 164 (1999).

00BowBowyer, S., Drake, J.J., Vennes, S.: Annu. Rev. Astron. Astrophys. 38 (2000) 231.

00MooMoos, H.W., Cash, W.C., Cowie, L.L., et al.: Astrophys. J. 538 (2000) L1.

00Sah Sahnow, D.J., Moos, H.W., Ake, T.B., et al.: Astrophys. J. 538 (2000) L7.

01MasMason, K.O., Breeveld, A., Much, R., et al.: Astron. Astrophys. 365 (2001) L36.

01MorMorales, C., Orozco, V., Gómez, J.F.: Astrophys. J. 552 (2001) 552.

01MurMurthy, J., Henry, R.C., Paxton, L.J., Proce, S.D.: Bull. Astr. Soc. India 29 (2001) 563.

01Str Strüder, L., Briel, U., Dennerl, K.: Astron. Astrophys. 365 (2001) L18.

02Gre Gregorio, A., Stalio, R., Broadfoot, L., Castelli, F., Hack, M., Holberg, J.: Astron. Astrophys. 383 (2002) 881.

02Wei Weisskopf, M.C., Brinkman, B., Canizares, C., et al.: Publ. Astron. Soc. Pacific 114 (2002) 1.

03Bar Barstow, M.A., Holberg, J.B.: Extreme Ultraviolet Astronomy, Cambridge University Press (2003).

03Hur Hurwitz, M.: Proc. SPIE 5164 (2003) 24.

04GehGehrels, N., Chincarini, G., Giommi, P., et al.: Astrophys. J. 611 (2004) 1005.

04MooMoos, H.W., McCandliss, S.R., Kruk, J.W.: SPIE 5488 (2004) 1.

04NewNewcomer, R.E., Murthy, J., Henry, R.C., Price, S.D., Paxton, L.: Air Force Research Laboratory Technical Report AFRL-VS-TR-2004-1056 (2004). Available as VizieR On-line Data Catalog II/269: MSX Ultraviolet Point Source Catalog.

05Hur Hurwitz, M., Sasseen, T.P., Sirk, M.M.: Astrophys. J. 623 (2005) 911.

05MarMartin, D.C., Fanson, J., Schiminovich, D.: Astrophys. J. 619 (2005) L1.

05MorMorissey, P., Schiminovich, D., Barlow, T.A., et al.: Astrophys. J. 619 (2005) L7.

06Ede Edelstein, J., Korpela, E.J., Adolfo, J., et al.: Astrophys. J. 644 (2006) L159.

07Val Valcu, B., Smith, P.L., Gardner, L.D., Raymond, J.C., Miralles, M.-P., Kohl, J.L.: Solar Phys. 243 (2007) 93.

08Pfe Pfeffermann, E.: The Universe in X-Rays, eds. J.E. Trümper, G. Hasinger, Springer (2008) 21.

09Tov Tovmassian, H.M.: priv. comm. (2009).

09Hol Holberg, J.B.: priv. comm. (2009).

09Hst HST webpage http://www.stsci.edu/hst/HST_overview/ at Space Telescope Science Institute (2009). 